\title{
Avaliação da qualidade de informação na internet: websites sobre o uso do Sistema de Frequência Modulada
}

\section{Evaluation of the quality of information on the internet: websites on the use of the Frequency Modulation System}

Vanessa Luisa Destro Fidêncio $^{1}$ (D), Dalva Maria Batista de Souza ${ }^{1}$ (D), Najla Ayanne Moura de Pinho ${ }^{1}$ (D), Roseane Silva Almeida ${ }^{1}$ (D), Joice de Moura Silva ${ }^{2}$ (D), Camila de Castro Corrêa ${ }^{1,3}$ (D)

\section{RESUMO}

Objetivo: avaliar a qualidade dos websites de maior acesso, voltados à orientação de professores sobre Sistemas de Frequência Modulada, em termos de legibilidade, confiabilidade, usabilidade e abrangência. Métodos: os websites contidos nas primeiras cinco páginas do Google e Yahoo!, obtidos mediante busca das palavras-chave/termos livres "sistema FM", "criança", "deficiência auditiva", "professor", "microfones remotos" e "orientações", foram selecionados e avaliados por três juízas, por meio do Teste de Facilidade de Leitura de Flesch, do Discern Questionnaire, da System Usability Scale e da avaliação de abrangência. Resultados: quatorze websites foram avaliados. Destes, metade foi classificada quanto ao grau de legibilidade e confiabilidade como "difícil/muito difícil" e "ruim", respectivamente. Quanto à usabilidade, a maioria dos webistes foi classificada como "boa", sendo apenas um categorizado como "melhor alcançável". A análise da abrangência dos websites revelou que os temas contemplados de forma menos precisa foram: a utilização do Sistema FM pelo professor e aluno e o manuseio do aparelho com o modo "liga/desliga". Conclusão: os websites disponíveis no idioma português do Brasil, com orientações para professores a respeito do Sistema FM, apresentaram legibilidade restrita e quase metade dos conteúdos disponibilizados foi classificada como "ruim", demonstrando que a confiabilidade para escolhas de tratamento por meio dessas informações não é tão segura.

Palavras-chave: Internet; Telemedicina; Tecnologia educacional; Fonoaudiologia; Equipamentos de autoajuda

\begin{abstract}
Purpose: To evaluate the quality of the most accessed websites aimed at instructing teachers on Frequency Modulation Systems in terms of readability, reliability, usability and comprehensiveness. Methods: The websites contained in the first five pages of Google and Yahoo!, obtained by searching for keywords / free terms: "FM system", "child", "hearing impairment", "teacher", "remote microphones" and " guidelines" were selected and evaluated by three analysts using the Flesch Reading EaseTest, the Discern Questionnaire, the System Usability Scale and the comprehensiveness assessment. Results: Fourteen websites were evaluated. Half of them were classified according to the degree of readability and reliability as "difficult / very difficult" and "poor", respectively. As for usability, most websites were classified as "good", with only one categorized as "best achievable". The analysis of the scope of the websites revealed that the themes covered in a less precise way were: the use of the FM system by the teacher and student, and the handling of the device with the "on / off" mode. Conclusion: The websites available in the Brazilian Portuguese language with teachers' guidance, regarding the FM System, presented restricted readability and almost half of the available content was classified as poor, demonstrating that the reliability for treatment choices through this information is not so safe.
\end{abstract}

Keywords: Internet; Telemedicine; Educational technology; Speech, Language and Hearing Sciences; Self-help devices

Trabalho realizado no Centro Universitário Planalto do Distrito Federal - UNIPLAN - Brasília (DF), Brasil.

${ }^{1}$ Centro Universitário Planalto do Distrito Federal - UNIPLAN - Brasília (DF), Brasil.

${ }^{2}$ Faculdade de Odontologia de Bauru, Universidade de São Paulo - FOB/USP - Bauru (SP), Brasil.

${ }^{3}$ Universidade de Brasília - UnB - Brasília (DF), Brasil.

Conflitos de interesse: Não.

Contribuições dos autores: VLDF, DMBS, NAMP, RSA e CCC participaram da concepção e delineamento do estudo, coleta, análise, interpretação dos dados e elaboração do artigo; VLDF, JMS e CCC colaboraram com a revisão crítica para conteúdo intelectual relevante e aprovação final da versão a ser apresentada para publicação.

Financiamento: Nada a declarar.

Autor correspondente: Vanessa Luisa Destro Fidêncio. E-mail: vanessa.destrof@gmail.com

Recebido: Março 17, 2021; Aceito: Maio 15, 2021 


\section{INTRODUÇÃO}

Crianças e jovens com deficiência auditiva, usuários de aparelho de amplificação sonora individual (AASI) e/ou implante coclear (IC), podem apresentar dificuldades em situações de ruído competitivo, por exemplo, no ambiente escolar, fazendo-se necessárias adaptações para tais contextos. Essas dificuldades implicam barreiras que impactam sobre as capacidades de reconhecimento e de compreensão auditiva da fala. Uma possibilidade facilitadora para esses indivíduos é o uso de microfones remotos, como o Sistema de Frequência Modulada $(\mathrm{FM})^{(1)}$. O Sistema FM consiste em um recurso utilizado para amenizar os efeitos do ruído, distância e reverberação no ambiente escolar, auxiliando na percepção auditiva da fala. $\mathrm{O}$ dispositivo funciona no modo sem fio, por meio de um transmissor com microfone, que fica com o professor/interlocutor e um receptor conectado ao AASI e/ou IC do aluno, permitindo a melhor compreensão da fala do interlocutor ${ }^{(2)}$.

A Portaria $n^{0} 1.274$ de 25 de junho de $2013^{(3)}$ garante, por meio do Sistema Único de Saúde (SUS), a concessão desse dispositivo para pessoas com deficiência auditiva usuárias de AASI e/ou IC. Dentre os critérios de indicação, destacam-se a exigência de que o indivíduo tenha entre 5 e 17 anos e 11 meses de idade e que esteja matriculado no ensino fundamental ou ensino médio. Após atualização por meio da Portaria no 3 de 19 de fevereiro de $2020^{(4)}$, a concessão do Sistema FM, no âmbito do SUS, foi ampliada para indivíduos com deficiência auditiva de qualquer idade, matriculados em todos os níveis acadêmicos.

No geral, os professores de escolas regulares desconhecem a função, modo de funcionamento e manuseio do Sistema $\mathrm{FM}^{(5)}$. Considerando que tais profissionais são peças fundamentais para a efetividade do uso desse dispositivo em sala de aula, é essencial que tenham informações e habilidades necessárias para sua utilização e manuseio de forma eficaz ${ }^{(2)}$.

Informações transmitidas via websites são de grande valia para a capacitação de profissionais da área da saúde ${ }^{(6)}$ e da educação(7), bem como para a orientação de familiares de crianças com deficiência auditiva ${ }^{(7,8)}$. Entretanto, somente a disponibilização dessas informações não é suficiente. É fundamental que os conteúdos online sejam provenientes de fontes confiáveis e com fundamentação na medicina baseada em evidências científicas, a fim de evitar a propagação de noções equivocadas/desatualizadas ${ }^{(9-11)}$.

Paralelamente à qualidade do conteúdo das informações, aponta-se para o cuidado quanto à legibilidade empregada na transmissão dos textos. Para atender tal questão, é primordial a utilização adequada de palavras, expressões e termos técnicos, tendo em vista a facilitação da leitura e da compreensão dos materiais apresentados, considerando a pluralidade do público leitor, que varia em idade, nível socioeconômico e escolaridade ${ }^{(8,12)}$.

Especificamente na área da Fonoaudiologia, poucos estudos foram realizados no sentido de averiguar a qualidade da informação disponibilizada na internet. Dentre eles, ressalta-se o trabalho que buscou quais informações estiveram disponíveis sobre as funções orofaciais ${ }^{(13)}$, disfagia orofaríngea ${ }^{(14)}$ e cirurgia de adenotonsilectomia ${ }^{(15)}$, o que se faz importante, uma vez que os websites mais bem avaliados pelos internautas e mais frequentemente acessados nem sempre apresentam as informações mais adequadas.

O atual contexto de crise de saúde pública e distanciamento social, desencadeado no ano de 2020 , provocado pela pandemia derivada da COVID-19 (SARS-CoV-2), resultou em uma série de mudanças sociais e enfatizou comportamentos humanos preexistentes, por exemplo, o uso das tecnologias digitais de informação e comunicação. $\mathrm{O}$ aumento do contato da população com diversos meios eletrônicos e mídias online, associado à dificuldade de acesso aos serviços de saúde de forma presencial, resultou em um cenário de maior procura/busca independente por informações em saúde nos mais variados assuntos, com vistas a suprir as necessidades, do ponto de vista dos pacientes, pais e profissionais impedidos de sanar as dúvidas e preocupações com profissionais especializados em acompanhamentos presenciais de rotina, temporariamente suspensos ${ }^{(16,17)}$. Tal realidade reforça a preocupação com a qualidade e a legibilidade dos materiais dispostos em websites.

Tendo em vista a escassez de estudos na área da Fonoaudiologia sobre a qualidade de websites, bem como a inexistência de pesquisas sobre a análise dos websites brasileiros sobre o Sistema FM, o objetivo deste estudo foi avaliar a qualidade dos websites de maior acesso, voltados à orientação sobre o Sistema FM para professores, em termos de legibilidade, confiabilidade, usabilidade e abrangência.

\section{MÉTODOS}

A presente pesquisa foi elaborada em duas etapas, sendo a primeira referente à busca dos websites direcionados à orientação de professores sobre uso do Sistema FM, com maior índice de acessos, e a segunda, sobre a avaliação da qualidade, dos princípios éticos, da legibilidade, da abrangência e da usabilidade dos websites. Por não analisar diretamente o impacto dos websites no público, o estudo não envolveu seres humanos, dispensando análise por comitê de ética e aplicação de Termo de Consentimento Livre e Esclarecido (TCLE).

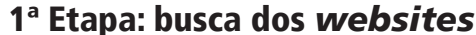

A procura por websites ocorreu durante o primeiro semestre do ano de 2020. As ferramentas de busca adotadas foram os sites de pesquisa Google e Yahoo!, considerados os buscadores mais utilizados em nível mundial. Nesta etapa, foram consultadas as cinco primeiras páginas originadas em cada uma das buscas.

Para localização dos websites, foram utilizadas as palavraschave/termos livres: "Sistema FM", "criança", "deficiência auditiva", "professor", "microfones remotos" e "orientações", de acordo com as combinações descritas no Quadro 1.

Como critérios de inclusão, foram admitidos websites, portais e blogs organizados no idioma português do Brasil, que alocassem informações e orientações sobre o conceito do Sistema FM, os benefícios, o uso e o manuseio por professores.

Foram excluídos websites cujas fontes de informação tinham o objetivo de noticiar fatos ou compartilhar experiências pessoais, documentos (artigos científicos, monografias, dissertações, teses

Quadro 1. Combinação dos termos livres para as buscas

\begin{tabular}{|cc|}
\hline Combinação 1 & Sistema FM / criança/ deficiência auditiva \\
Combinação 2 & Sistema FM/ professor/ orientação \\
Combinação 3 & Sistema FM/ orientação \\
Combinação 4 & Sistema FM \\
Combinação 5 & microfones remotos/ criança/ deficiência auditiva \\
\hline
\end{tabular}


e recomendações), vídeos do YouTube, websites de notícias e fóruns online de discussão em grupo.

A busca e a seleção dos websites foram realizadas por três juízas, graduandas do curso de Fonoaudiologia. Antes da avaliação, todas receberam capacitação prévia sobre o Sistema FM por uma fonoaudióloga com dez anos de experiência na área e também foram definidos os critérios padrões considerados na análise dos conteúdos disponibilizados pelos websites. Em seguida, estabeleceu-se uma lista única de websites que contemplavam os critérios de inclusão e de elegibilidade. Nos casos de discordância, uma quarta juíza fonoaudióloga foi incluída para a revisão e o estabelecimento do consenso.

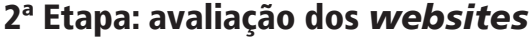

Os websites selecionados foram analisados de modo independente pelas três juízas, conforme três protocolos estabelecidos pela literatura: o Teste de Facilidade de Leitura de Flesch ${ }^{(18)}$, que investiga o nível de legibilidade por meio da fórmula do Índice de Facilidade de Leitura de Flesch (IFLF); o Discern Questionnaire (DQ) ${ }^{(19)}$ (Anexo 1), que avalia a qualidade dos conteúdos e a System Usability Scale (SUS) ${ }^{(20)}$ (Anexo 2), que mensura a usabilidade. Para todas as análises, foram consideradas somente as páginas específicas sobre o assunto.

O IFLF calcula, de forma automatizada, o nível de legibilidade, considerando o comprimento médio das sentenças e o número médio de sílabas por palavra. Trata-se de uma das fórmulas de legibilidade mais utilizadas pela literatura ${ }^{(21)}$. A pontuação 0 indica baixa legibilidade, enquanto 100 pontos revelam que o texto possui alto nível de legibilidade. Considera-se, para esta classificação a porcentagem 0-30\% como "muito difícil" (nível superior); 30-50\% "difícil" (nível médio/superior); 5060\% "razoavelmente difícil" (início do nível médio); 60-70\% "padrão" (7 $7^{\mathrm{a}}$ e $8^{\mathrm{a}}$ série); 70-80\% "razoavelmente fácil" (6 $6^{\mathrm{a}}$ série); 80-90\% "fácil" ( $5^{a}$ série) e 90-100\% "muito fácil" (4 série) ${ }^{(21)}$.

O DQ é composto por 16 perguntas. Da pergunta 1 até a 15 , é avaliada a confiabilidade das informações nas escolhas de tratamento de saúde, enquanto a $16^{\mathrm{a}}$ questão avalia a pontuação geral da qualidade das informações. Para calcular o resultado do teste, as questões foram pontuadas de 1 a 5 e, quanto maior a pontuação, melhor a avaliação do conteúdo online. Cada pergunta contém informações discriminadas para facilitar a avaliação. Essa ferramenta é dividida em cinco formas de pontuação: de 16-26, a resposta é classificada como "muito ruim"; entre 27-38, "ruim"; 39-50, "razoável"; 51-62, "boa" e maior que 63 pontos, "excelente" (22).

A escala SUS é constituída por dez questões afirmativas, referentes à usabilidade do objeto avaliado. As afirmações ímpares indicam os pontos positivos, enquanto as afirmações pares indicam os pontos negativos na usabilidade. Para calcular a média total da usabilidade, são somados os valores de cada item, que podem variar entre 0 e 5 , classificados como "discordo fortemente", "discordo", "não concordo nem discordo", "concordo" e "concordo fortemente". O valor de escore da escala SUS varia de 0 a 100 , sendo de $0-25$ considerado "o pior alcançável"; de 25-39, "ruim"; 39-52, "aceitável”; 52-74, "bom"; 74-85, excelente e de 85-100, "o melhor alcançável"(23).

Para análise da abrangência de cada website, os juízes observaram se os conteúdos contemplavam, ou não, informações pertinentes à orientação do professor sobre quatro temas centrais, previamente selecionados: 1- definição do Sistema FM; 2- importância do uso do dispositivo em sala de aula; 3- modo de utilização pelo professor e pelo aluno: posicionamento do transmissor (microfone) e receptor; 4- manuseio do transmissor pelo professor: ligar/desligar, opção de "microfone mudo". Com base na concordância dos padrões de respostas entre os juízes, foram atribuídas as seguintes pontuações: 1 ponto, "muito insuficiente"; 2 , "insuficiente"; 3 , "regular"; 4, "satisfatório" e 5 pontos, "muito satisfatório". A abrangência dos websites foi definida a partir da média da pontuação de cada quesito avaliado.

Os resultados foram tabulados e submetidos à análise quantitativa descritiva dos dados, por meio da média e do desvio padrão. Após, realizou-se a comparação dos parâmetros adotados, a fim de estipular um ranking geral de qualidade entre os websites voltados à orientação de professores sobre o Sistema FM, disponíveis no idioma português do Brasil.

\section{RESULTADOS}

Dos 500 websites encontrados nas plataformas de busca (250 Google e 250 Yahoo!), 480 foram excluídos e 20 websites (12 Google e oito Yahoo!) foram selecionados. Entretanto, no decorrer do estudo 6 websites deixaram de estar disponíveis. Portanto, a amostra foi composta por 14 websites. O fluxograma referente às etapas de busca e seleção encontra-se descrito na Figura 1.

A Tabela 1 registra os aspectos de legibilidade mensurados por meio do IFLF.

Os dados referentes à confiabilidade, analisados pelo questionário DQ, são apresentados nas Tabelas 2 e 3.

Na Tabela 4, está disposto o total das notas atribuídas à usabilidade, a partir da escala SUS.

A análise da abrangência dos websites encontra-se na Tabela 5.

O Quadro 2 apresenta um panorama global da análise dos websites, no que diz respeito às maiores e menores pontuações obtidas nas avaliações aplicadas.

\section{DISCUSSÃO}

Os websites são ferramentas utilizadas por diversos tipos de leitores como fonte de busca, quando procuram uma determinada informação. No último ano, principalmente em decorrência da pandemia derivada da COVID-19 (SARS-CoV-2), observouse um aumento no uso das mídias e da internet por adultos ${ }^{(17)}$. Isso porque as novas estratégias pedagógicas, de saúde, sociais, entre outras, implantadas pela sociedade atual do "novo normal" transformaram-se, na grande maioria, em modalidades remota ${ }^{24}$. Por isso, a avaliação das fontes de informação disponíveis em formato online é de fundamental importância para que sejam capazes de tornarem-se aliadas às medidas de promoção e de educação em saúde. Por exemplo, a garantia da disponibilização de materiais de qualidade em websites possibilita o uso e a indicação dos profissionais de saúde como meio de complementação das informações fornecidas em suas consultas.

Apesar da procura realizada em dois grandes sites de buscas, do total de 500 websites, somente $14(2,8 \%)$ englobaram a temática do Sistema FM (Figura 1). Em outros estudos que utilizaram as mesmas ferramentas de busca em relação às funções orofaciais, de 350 websites, 35 (10\%) atenderam aos critérios de pesquisa para inclusão ${ }^{(13)}$. Na temática disfagia 


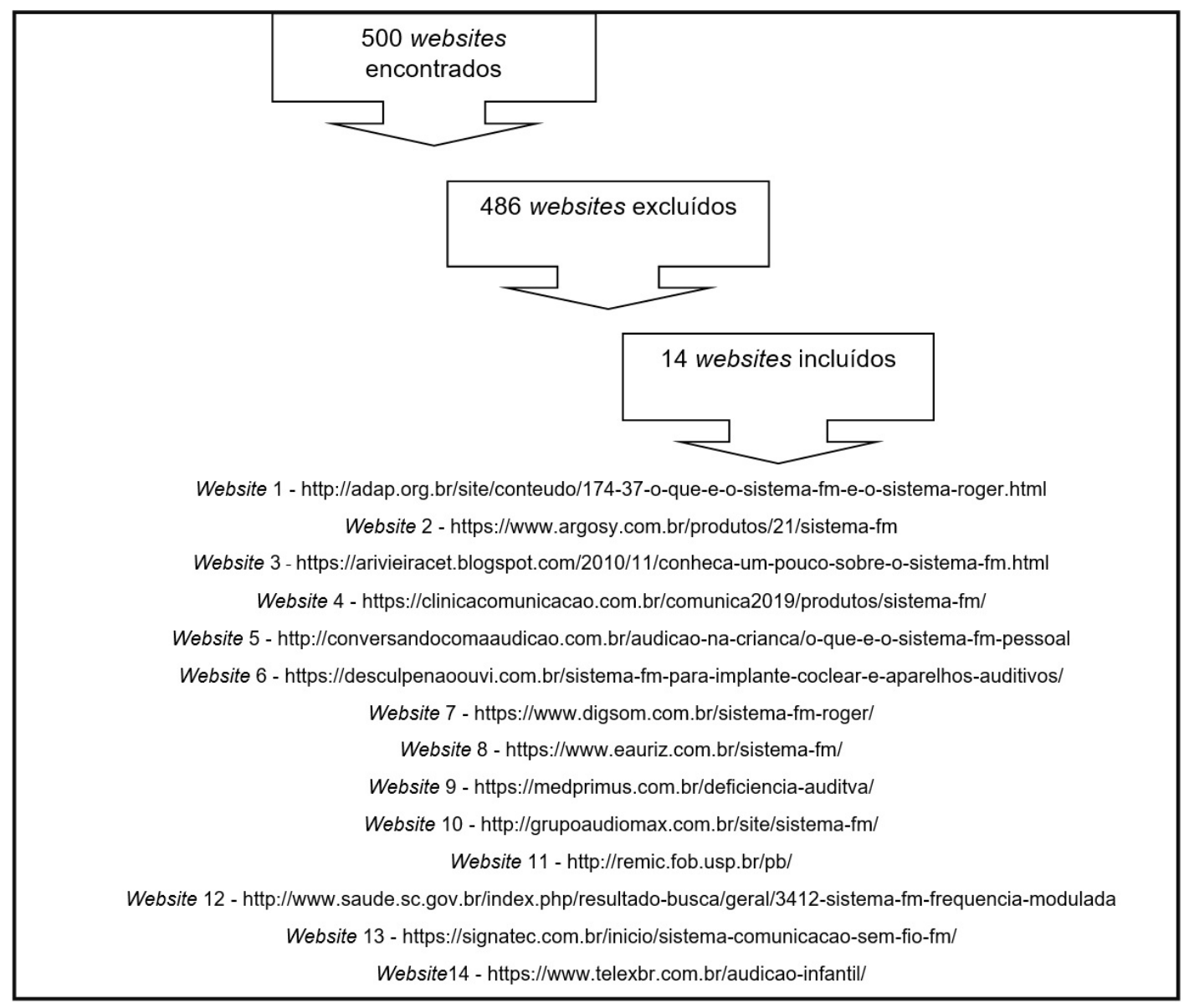

Figura 1. Fluxograma da pesquisa realizada sobre os websites do sistema FM

Tabela 1. Análise da legibilidade dos websites disponíveis no idioma português brasileiro sobre o Sistema de Frequência Modulada, por meio da aplicação do Índice de Facilidade de Leitura de Flesch

\begin{tabular}{ccc}
\hline WEBSITE & LEGIBILIDADE DO IFLF & CLASSIFICAÇÃO \\
\hline 1 & $53,73 \%$ & Razoavelmente difícil \\
2 & $57,23 \%$ & Razoavelmente difícil \\
3 & $70,29 \%$ & Razoavelmente fácil \\
4 & $37,03 \%$ & Difícil \\
5 & $33,40 \%$ & Difícil \\
6 & $44,93 \%$ & Difícil \\
7 & $45,47 \%$ & Difícil \\
8 & $51,36 \%$ & Razoavelmente difícil \\
9 & $46,05 \%$ & Difícil \\
10 & $87,72 \%$ & Fácil \\
11 & $59,06 \%$ & Razoavelmente difícil \\
12 & $18,82 \%$ & Muito difícil \\
13 & $58,30 \%$ & Razoavelmente difícil \\
14 & $43,84 \%$ & Difícil \\
\hline
\end{tabular}

Legenda: IFLF = Índice de Facilidade de Leitura de Flesch

orofaríngea no idoso, foram selecionados 19 (9,5\%) websites dos 200 identificados durante as buscas ${ }^{(14)}$. No que se refere à orientação de leigos em cirurgia de adenotonsilectomia, foram considerados 34 (34\%) websites dos 100 elegidos de acordo com os critérios exigidos ${ }^{(15)}$. Tais dados reforçam a escassez de conteúdos disponibilizados em formato online a respeito do uso do Sistema FM.
No quesito legibilidade, $50 \%$ da amostra dos websites localizados foram classificados como "difíceis/muito difíceis" (Tabela 1), ou seja, metade dos websites requer dos leitores um nível superior de estudo para sua legibilidade, o que limita o acesso à informação. Comparando a outros estudos que utilizaram o mesmo meio de avaliação, observou-se um nível de dificuldade de $47,8 \%$ na pesquisa com o tema cirurgia de adenotonsilectomia ${ }^{(15)}$ e de $61,23 \%$ no tema de funções orofaciais, classificando os materiais como apropriados. Ressalta-se que o teste de legibilidade apresenta algumas restrições, uma vez que a avaliação é pouco profunda e leva em conta somente a contagem de sílabas e palavras e não o conteúdo apresentado ${ }^{(25)}$.

Quanto à confiabilidade, a maioria dos websites incluídos neste estudo (42,85\%) foi classificada como "ruim" (Tabela 3). Tal achado vai de encontro aos dados obtidos em outra pesquisa que propôs a avaliação da confiabilidade e observou que nenhum dos websites atingiu a classificação "excelente" ou "muito ruim". Entre as justificativas para tal achado, os autores relataram que em $60 \%$ dos websites, as referências não foram fornecidas, o que confirmou a baixa qualidade da informação ${ }^{(22)}$.

A média geral da escala SUS foi de 70,0 pontos, sendo atribuída a classificação de website "bom" (Tabela 4). A mesma classificação foi obtida em um estudo que utilizou esse protocolo para a análise de usabilidade de um sistema de informação de saúde neonatal, segundo a percepção dos usuários ${ }^{(26)}$.

No que diz respeito à abrangência, observou-se que, em grande parte dos websites incluídos, as informações foram classificadas como "insuficientes" nos critérios de "modo de 
닌

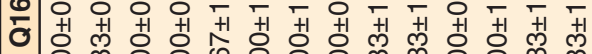

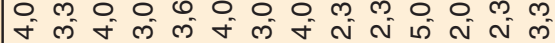
৪

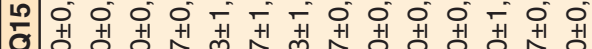

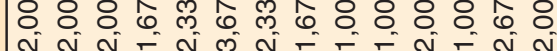

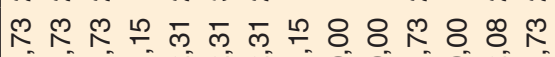

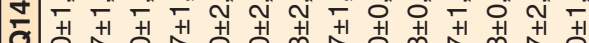

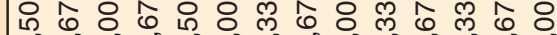
mi

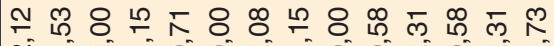

m ల్ల ల్ల ల్ల ల్ల ల్ల ల్లి

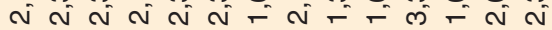

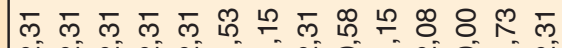

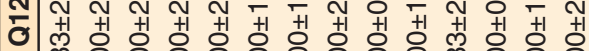
ल्m 8 o 8 o 8 o 8 o 8 o ৪৪৪৪৪৪৪৪৪৪

ᄃ。 mir r r r r i r i

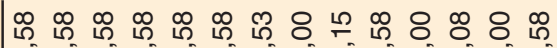

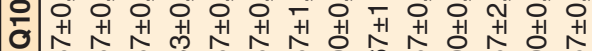

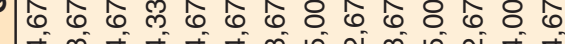
గొ

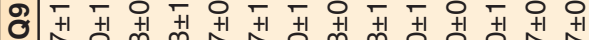

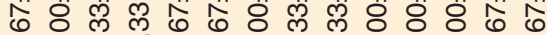
त ल m n m m m ஃ ৪ ৪ ৪

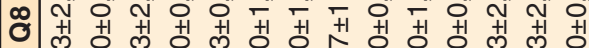

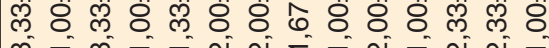

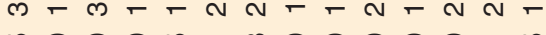
느유.

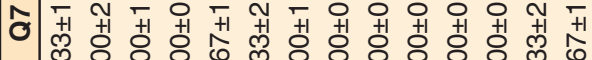

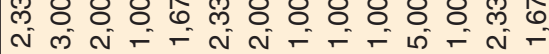
ఐ న

8 草

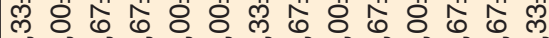
m ल

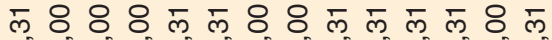

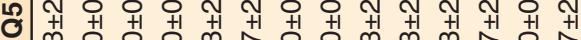

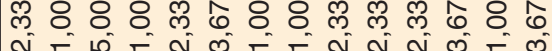
ஃ ஃ

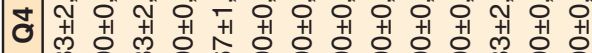

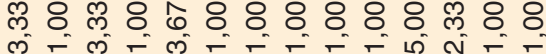
৪ ํำ

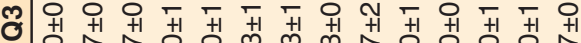

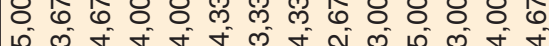
৪ ৪ 유

ช

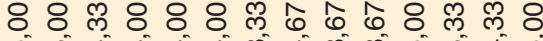
एल

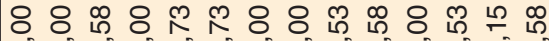

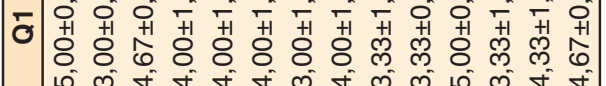
ш - 
utilização pelo professor e pelo aluno: posicionamento do transmissor (microfone) e receptor"; "manuseio do transmissor pelo professor: ligar/desligar, opção de "microfone mudo" (Tabela 5). Este resultado concorda com o estudo supracitado ${ }^{(14)}$

Tabela 3. Análise da média total e classificação da avaliação pelo Discern Questionnaire

\begin{tabular}{ccc}
\hline WEBSITES & TOTAL DQ & CLASSIFICAÇÃO \\
\hline 1 & 54,15 & Bom \\
2 & 37,67 & Ruim \\
3 & 53,33 & Bom \\
4 & 37,00 & Ruim \\
5 & 48,17 & Regular \\
6 & 50,67 & Regular \\
7 & 36,99 & Ruim \\
8 & 43,34 & Regular \\
9 & 30,66 & Ruim \\
10 & 35,00 & Ruim \\
11 & 60,66 & Bom \\
12 & 34,66 & Ruim \\
13 & 42,33 & Regular \\
14 & 45,01 & Regular \\
\hline
\end{tabular}

Legenda: DQ = Discern Questionnaire sobre disfagia orofaríngea no idoso, no qual a avaliação da abrangência foi de 33,6\%.

O website11 (Web11) foi o melhor pontuado no que diz respeito à confiabilidade, usabilidade e abrangência (Quadro 2). Foi idealizado por uma instituição pública de ensino superior, o que pode explicar a qualidade da informação disponibilizada. Foi o único website a atingir a classificação de "melhor alcançável" na escala SUS e pontuação máxima nas questões relativas à abrangência do conteúdo. No entanto, não apresentou pontuação tão elevada no quesito legibilidade, sendo classificado como "razoavelmente difícil" nesse aspecto.

Apesar de não substituir as orientações realizadas por um fonoaudiólogo especializado, não há como evitar que os professores busquem, por conta própria, informações a respeito do uso do Sistema FM, disponíveis em formato online. Assim, torna-se essencial o oferecimento de informação de qualidade, de modo a auxiliá-los na inclusão dos alunos usuários desse dispositivo em sala de aula. Nesse sentido, a avaliação da informação em saúde disponibilizada online é de extrema importância, principalmente nos tempos atuais, pois é uma questão de saúde pública garantir que os websites disponíveis sejam críveis e tragam segurança ao cidadão usuário, seja ele paciente, familiar ou profissional ${ }^{(27)}$.

Tabela 4. Resultado da análise do questionário de usabilidade System Usability Scale

\begin{tabular}{|c|c|c|c|c|c|c|c|c|c|c|c|c|}
\hline WEBSITES & Q1 & Q2 & Q3 & Q4 & Q5 & Q6 & Q7 & Q8 & Q9 & Q10 & TOTAL & CLASSIFICAÇÃO \\
\hline 1 & $3,33 \pm 0,71$ & $4,00 \pm 2,12$ & $3,67 \pm 0,71$ & $2,33 \pm 0,23$ & $3,00 \pm 0,71$ & $3,33 \pm 1,18$ & $4,00 \pm 0,71$ & $3,33 \pm 1,18$ & $3,67 \pm 0,71$ & $1,00 \pm 2,12$ & 79,17 & Excelente \\
\hline 2 & $2,67 \pm 0,71$ & $4,00 \pm 2,12$ & $3,67 \pm 0,71$ & $2,33 \pm 0,23$ & $3,00 \pm 0,71$ & $2,00 \pm 0,71$ & $3,33 \pm 0,71$ & $3,33 \pm 1,18$ & $3,33 \pm 0,71$ & $1,00 \pm 2,12$ & 71,67 & Bom \\
\hline 3 & $3,00 \pm 0,71$ & $4,00 \pm 2,12$ & $3,67 \pm 0,71$ & $2,33 \pm 0,23$ & $3,33 \pm 0,71$ & $3,00 \pm 0,71$ & $3,67 \pm 0,71$ & $3,00 \pm 0,71$ & $3,67 \pm 0,71$ & $1,00 \pm 2,12$ & 76,67 & Excelente \\
\hline 4 & $2,00 \pm 0,71$ & $3,67 \pm 1,65$ & $2,67 \pm 0,71$ & $2,33 \pm 0,23$ & $3,00 \pm 0,71$ & $2,67 \pm 0,24$ & $3,67 \pm 0,71$ & $4,00 \pm 2,12$ & $3,33 \pm 0,71$ & $1,00 \pm 2,12$ & 70,83 & Bom \\
\hline 5 & $3,33 \pm 0,71$ & $3,33 \pm 1,18$ & $3,67 \pm 0,71$ & $2,33 \pm 0,23$ & $3,33 \pm 0,71$ & $2,67 \pm 0,24$ & $3,67 \pm 0,71$ & $4,00 \pm 2,12$ & $3,33 \pm 0,71$ & $1,33 \pm 1,65$ & 77,50 & Excelente \\
\hline 6 & $3,33 \pm 0,71$ & $3,33 \pm 1,18$ & $2,00 \pm 0,71$ & $2,67 \pm 0,23$ & $3,67 \pm 0,71$ & $2,67 \pm 0,24$ & $4,00 \pm 0,71$ & $4,00 \pm 2,12$ & $4,00 \pm 0,71$ & $1,00 \pm 2,12$ & 76,67 & Excelente \\
\hline 7 & $1,67 \pm 0,71$ & $4,00 \pm 2,12$ & $2,67 \pm 0,71$ & $2,33 \pm 0,23$ & $3,67 \pm 0,71$ & $2,67 \pm 0,24$ & $3,67 \pm 0,71$ & $3,33 \pm 1,18$ & $3,00 \pm 0,71$ & $0,67 \pm 2,59$ & 69,17 & Bom \\
\hline 8 & $2,67 \pm 0,71$ & $3,67 \pm 1,65$ & $4,00 \pm 0,71$ & $2,33 \pm 0,23$ & $3,33 \pm 0,71$ & $2,67 \pm 0,24$ & $3,67 \pm 0,71$ & $4,00 \pm 2,12$ & $3,67 \pm 0,71$ & $1,00 \pm 2,12$ & 77,50 & Excelente \\
\hline 9 & $1,00 \pm 0,71$ & $3,67 \pm 1,65$ & $0,33 \pm 0,71$ & $2,00 \pm 0,70$ & $2,33 \pm 0,71$ & $3,00 \pm 0,71$ & $3,67 \pm 0,71$ & $4,00 \pm 2,12$ & $2,67 \pm 0,71$ & $0,67 \pm 2,59$ & 58,33 & Bom \\
\hline 10 & $1,33 \pm 0,71$ & $3,67 \pm 1,65$ & $1,33 \pm 0,71$ & $2,00 \pm 0,70$ & $2,33 \pm 0,71$ & $3,00 \pm 0,71$ & $3,00 \pm 0,71$ & $3,67 \pm 1,65$ & $2,67 \pm 0,71$ & $1,00 \pm 2,12$ & 60,00 & Bom \\
\hline 11 & $4,00 \pm 0,71$ & $4,00 \pm 1,18$ & $3,67 \pm 0,71$ & $3,67 \pm 0,70$ & $4,00 \pm 0,71$ & $2,67 \pm 0,71$ & $3,67 \pm 0,71$ & $4,00 \pm 0,71$ & $4,00 \pm 0,71$ & $1,33 \pm 2,12$ & 87,50 & $\begin{array}{l}\text { Melhor } \\
\text { alcançável }\end{array}$ \\
\hline 12 & $1,00 \pm 0,71$ & $3,67 \pm 1,65$ & $2,00 \pm 0,71$ & $2,33 \pm 0,23$ & $1,33 \pm 0,71$ & $3,67 \pm 1,65$ & $0,67 \pm 0,71$ & $3,67 \pm 1,65$ & $2,00 \pm 0,71$ & $1,67 \pm 1,18$ & 55,00 & Bom \\
\hline 13 & $0,67 \pm 0,71$ & $3,67 \pm 1,65$ & $2,00 \pm 0,71$ & $2,00 \pm 0,70$ & $1,33 \pm 0,71$ & $3,67 \pm 1,65$ & $2,00 \pm 0,71$ & $3,67 \pm 1,65$ & $2,00 \pm 0,71$ & $1,67 \pm 1,18$ & 56,67 & Bom \\
\hline 14 & $2,00 \pm 0,71$ & $3,67 \pm 1,65$ & $1,33 \pm 0,71$ & $2,33 \pm 0,23$ & $3,33 \pm 0,71$ & $2,67 \pm 0,24$ & $2,67 \pm 0,71$ & $3,67 \pm 1,65$ & $3,00 \pm 0,71$ & $1,33 \pm 1,65$ & 65,00 & Bom \\
\hline $\begin{array}{l}\text { MÉDIA } \\
\text { GERAL }\end{array}$ & & & & & & & & & & & 70,12 & Bom \\
\hline
\end{tabular}

Tabela 5. Resultado da média da avaliação sobre abrangência a respeito das informações contidas nos websites

\begin{tabular}{ccccrr}
\hline WEBSITES & DEFINIÇÃO & $\begin{array}{c}\text { IMPORTÂNCIA EM } \\
\text { SALA DE AULA }\end{array}$ & $\begin{array}{c}\text { UTILIZAÇÃO PELO } \\
\text { PROFESSOR E ALUNO }\end{array}$ & LIGA/DESLIGA & ABRANGÊNCIA GERAL \\
\hline 1 & $4,33 \pm 0,58$ & $4,33 \pm 0,58$ & $3,00 \pm 1,00$ & $1,00 \pm 0,00$ & 12,66 \\
2 & $4,00 \pm 0,00$ & $2,33 \pm 0,58$ & $1,67 \pm 1,15$ & $1,33 \pm 0,00$ & 9,33 \\
3 & $4,67 \pm 0,58$ & $4,67 \pm 0,58$ & $4,33 \pm 0,58$ & $1,00 \pm 0,00$ & 14,67 \\
4 & $4,00 \pm 0,00$ & $2,67 \pm 1,15$ & $1,00 \pm 0,00$ & $1,00 \pm 0,00$ & 8,67 \\
5 & $3,67 \pm 2,31$ & $5,00 \pm 0,00$ & $4,67 \pm 0,58$ & $1,00 \pm 0,00$ & 14,34 \\
6 & $4,00 \pm 1,73$ & $4,67 \pm 0,58$ & $5,00 \pm 0,00$ & $1,00 \pm 0,00$ & 14,67 \\
7 & $3,00 \pm 1,00$ & $2,33 \pm 1,53$ & $3,67 \pm 0,58$ & $1,00 \pm 0,00$ & 10,00 \\
8 & $4,33 \pm 0,58$ & $4,00 \pm 1,00$ & $5,00 \pm 0,00$ & $1,00 \pm 0,00$ & 14,33 \\
9 & $2,00 \pm 1,00$ & $2,67 \pm 0,58$ & $3,67 \pm 2,31$ & $1,00 \pm 0,00$ & 9,34 \\
10 & $3,00 \pm 0,00$ & $2,33 \pm 0,58$ & $4,33 \pm 1,15$ & $1,33 \pm 0,58$ & 1,99 \\
11 & $5,00 \pm 0,00$ & $5,00 \pm 0,00$ & $5,00 \pm 0,00$ & $5,00 \pm 0,00$ & 2,00 \\
12 & $3,67 \pm 1,15$ & $2,33 \pm 1,15$ & $2,67 \pm 1,53$ & $1,00 \pm 0,00$ & 9,67 \\
13 & $4,00 \pm 0,00$ & $3,33 \pm 1,53$ & $2,67 \pm 2,08$ & $1,00 \pm 0,00$ & 1,00 \\
14 & $4,00 \pm 0,00$ & $4,33 \pm 0,58$ & $4,33 \pm 0,58$ & $1,00 \pm 0,00$ & 13,66 \\
\hline
\end{tabular}


Quadro 2. Análise geral das três melhores e três piores pontuações dos quatro testes avaliadores das informações sobre o Sistema de Frequência Modulada

\begin{tabular}{|c|c|c|c|c|}
\hline & IFLF & $\mathbf{D Q}$ & SUS & Abrangência \\
\hline \multirow[t]{3}{*}{ Maior pontuação } & Web10 (87,72\%) & Web11 $(60,66)$ & Web11 $(87,50)$ & Web11 (20) \\
\hline & Web3 $(70,29 \%)$ & Web1 $(54,15)$ & Web1 $(79,17)$ & Web3/6 $(14,67)$ \\
\hline & Web14 (57,23\%) & Web3 $(53,33)$ & Web5/8 $(77,50)$ & Web5 $(14,34)$ \\
\hline \multirow[t]{3}{*}{ Menor Pontuação } & Web12 (18,82\%) & Web9 $(30,66)$ & Web12 $(55,0)$ & Web4 $(8,67)$ \\
\hline & Web5 (33,40\%) & Web12 $(34,60)$ & Web13 $(56,67)$ & Web2 $(9,33)$ \\
\hline & Web4 (37,03\%) & Web10 $(35,0)$ & Web9 $(58,33)$ & Web9 $(9,34)$ \\
\hline
\end{tabular}

Legenda: IFLF = Índice de Facilidade de Leitura de Flesch; DQ = Discern Questionnaire; SUS = System Usability Scale; Web = website

Faz-se necessária a realização de novos estudos sobre a temática, com vistas a promover discussões que despertem nos profissionais criadores de conteúdos online o interesse e a preocupação, não só com a elaboração de materiais informativos confiáveis e baseados em evidências científicas, mas, também, comprometidos com uma transmissão de maneira acessível, no que diz respeito à legibilidade do conteúdo à pluralidade do público-alvo leitor.

\section{CONCLUSÃO}

Os websites disponíveis no idioma português do Brasil com orientações para professores a respeito do Sistema FM apresentaram legibilidade restrita, exigindo dos leitores um nível de escolaridade médio/superior para a compreensão dos conteúdos fornecidos. Além disso, quase metade dos conteúdos disponibilizados foi classificada como "ruim", demonstrando que a confiabilidade para escolhas de tratamento por meio dessas informações não é tão segura.

A maioria dos websites não contempla informações importantes sobre o uso do Sistema FM pelo professor, em sala de aula.

\section{REFERÊNCIAS}

1. Barreiros GE, Mendes BCA, Ficker LB, Novaes BCAC. Reabilitação auditiva: papel da família na adesão ao uso do sistema de transmissão sem fio na escola. Distúrb Comun. 2016;28(4):718-29.

2. Carvalho DS, Pedruzzi CM. Uso do sistema de frequência modulada por escolares com perda auditiva. Distúrb Comun. 2019;31(1):12-21. http://dx.doi.org/10.23925/2176-2724.2019v31i1p12-21.

3. Brasil. Ministério da Saúde. Portaria $\mathrm{n}^{\circ} 1274$, de 25 de junho de 2013. Inclui o Procedimento de Sistema de Frequência Modulada Pessoal (FM) na Tabela de Procedimentos, Medicamentos, Órteses, Próteses e Materiais Especiais (OPM) do Sistema Único de Saúde. Diário Oficial da União [Internet]; 2013. [citado em 2021 Fev 20]. Disponível em: https://bvsms.saude.gov.br/bvs/saudelegis/gm/2013/ prt1274_25_06_2013.html

4. Brasil. Ministério da Saúde/ Secretaria de Ciência, Tecnologia, Inovação e Insumos Estratégicos em Saúde. Portaria no 3, de 19 de fevereiro de 2020. Torna pública a decisão de ampliar o uso do Sistema de Frequência Modulada Pessoal para indivíduos com deficiência auditiva de qualquer idade matriculados em qualquer nível acadêmico, no âmbito do Sistema Único de Saúde - SUS. Diário Oficial da União [Internet]; 2020. [citado em 2021 Fev 20]. Disponível em: https://www.in.gov. br/web/dou/-/portaria-n-3-de-19-de-fevereiro-de-2020-244302714

5. Fidêncio VLD, Costa CA, Sousa IS, Romão JMFE. Investigação do conhecimento de professores de escolas regulares de uma região do Distrito Federal sobre o sistema de frequência modulada. Audiol Commun Res. 2020;25:e2278. http://dx.doi.org/10.1590/2317-64312019-2278.

6. Maximino LP, Zambonato TCF, Picolini-Pereira MM, Corrêa CC, Feniman MR, Blasca WQ. Development and evaluation of a blog about cleft lip and cleft palate and hearing. Int Arch Otorhinolaryngol. 2018;22(1):60-7. http://dx.doi.org/10.1055/s-0037-1603494. PMid:29371900.

7. Corrêa CC, Silva RA, Blasca WQ. Elaboration and evaluation of contents about hearing health inserted in cybertutor. Int Arch Otorhinolaryngol. 2014;18(2):115-21. http://dx.doi.org/10.1055/s-0033-1358578. PMid:25992075.

8. Chaves JN, Libardi AL, Agostinho-Pesse RS, Morettin M, Alvarenga KF. Telessaúde: avaliação de websites sobre triagem auditiva neonatal na Língua Portuguesa. CoDAS. 2015;27(6):526-33. http://dx.doi. org/10.1590/2317-1782/20152014169. PMid:26691616.

9. Roshan A, Agarwal S, England RJ. Role of information available over the internet: what are the parents of children undergoing tonsillectomy likely to find? Ann R Coll Surg Engl. 2008;90(7):601-5. http://dx.doi. org/10.1308/003588408X318129. PMid:18701014.

10. Strychowsky JE, Nayan S, Farrokhyar F, MacLean J. YouTube: a good source of information on pediatric tonsillectomy? Int J Pediatr Otorhinolaryngol. 2013;77(6):972-5. http://dx.doi.org/10.1016/j. ijporl.2013.03.023. PMid:23598152.

11. Sorensen JA, Pusz MD, Brietzke SE. YouTube as na information source for pediatric adenotonsillectomyandeartube surgery. Int J Pediatr Otorhinolaryngol. 2014;78(1):65-70. http://dx.doi.org/10.1016/j. ijporl.2013.10.045. PMid:24315211.

12. Favoretto NC, Carleto NG, Arakawa AM, Alcalde MP, Bastos JRM, Caldana ML. Portal dos idosos: desenvolvimento e avaliação de um website com informações sobre o processo de envelhecimento e as principais alterações fonoaudiológicas que acometem os idosos. CoDAS. 2017;29(5):e20170066. http://dx.doi.org/10.1590/23171782/20172017066. PMid:29069273.

13. Corrêa CC, Ferrari DV, Berretin-Felix G. Quality, range, and legibility in web sites related to orofacial functions. Int Arch of Otorhinolaryngol. 2013;17(4):358-62.

14. Corrêa CC, Costa DR, Berretin-Felix G. Websites em português sobre disfagia orofaríngea no idoso. Rev Disturb Comun. 2018;30(1):140-6. http://dx.doi.org/10.23925/2176-2724.2018v30i1p140-146.

15. Piña SP, Corrêa CC, Carvalho LR, Weber SAT. Comprehensiveness, readability, and reliability of Brazilian websites available for lay people guidance on adenotonsillectomy. Braz J Otorhinolaryngol. 2021;87(1):66-73. http://dx.doi.org/10.1016/j.bjorl.2019.06.012. PMid:31439530.

16. Senhoras EM. Coronavírus e educação: análise dos impactos assimétricos. Revista UFRR. 2020;2(5):128-33. 
17. Malta DC, Szwarcwald CL, Barros MBA, Gomes CS, Machado IH, Souza PRB Jr, et al. A pandemia da COVID-19 e as mudanças no estilo de vida dos brasileiros adultos: um estudo transversal. Epidemiol Serv Saude. 2020;29(4):e2020407. http://dx.doi.org/10.1590/s167949742020000400026. PMid:32997069.

18. Flesch R. How to write plain English. New York, NY: Harper and Row; 1979

19. Charnock D, Shepperd S, Needham G, Gann R. DISCERN: an instrument for judging the quality of written consumer health information on treatment choices. J Epidemiol Community Health. 1999;53(2):10511. http://dx.doi.org/10.1136/jech.53.2.105. PMid:10396471.

20. Liang J, Xian D, Liu X, Fu J, Zhang X, Tang B, et al. Usability study of mainstream wearable fitness devices: feature analysis and system usability scale evaluation. JMIR Mhealth Uhealth. 2018;6(11):e11066. http://dx.doi.org/10.2196/11066. PMid:30409767.

21. Lyra DH, Amaral CLF. Apreensibilidade e legibilidade de artigos científicos de um periódico nacional. Tekhne e Logos. 2012;3(3):90101.

22. Olkun HK, Demirkaya AA. Evaluation of internet information about lingual orthodontics using DISCERN and JAMA Tools. Turk J Orthod.
2018;31(2):50-4. http://dx.doi.org/10.5152/TurkJOrthod.2018.17042. PMid:30112514.

23. Santos BM, Cordeiro MEC, Schneider IJC, Ceccon RF. Educação médica durante a pandemia da COVID-19: uma revisão de escopo. Rev Bras Educ Med. 2020;44(suppl.1):e0139.

24. Feitosa ANA, Nascimento IMG, Alencar RL Na, Alencar MT, Tavares MML, Assis EV. Tecnologias educacionais em tempos de pandemia: relato de experiência. Braz J Production Engineering. 2020;6(6):166-72.

25. Corrêa CC, Pauleto ARC, Ferrari DV, Berretin-Felix G. Website Babies Portal: development and evaluation of the contents regarding orofacial functions. J Appl Oral Sci. 2013;21(6):581-9. http://dx.doi. org/10.1590/1679-775720130267. PMid:24473726.

26. Padrini-Andrade L, Balda RCX, Areco KCN, Bandiera-Paiva P, Nunes MV, Marba STM, et al. Avaliação da usabilidade de um sistema de informação em saúde neonatal segundo a percepção do usuário. Rev Paul Pediatr. 2019;37(1):90-6. http://dx.doi.org/10.1590/19840462/;2019;37;1;00019. PMid:30569950.

27. Moretti FA, Oliveira VE, Silva EMK. Acesso a informações de saúde na internet: uma questão de saúde pública? Rev Assoc Med Bras. 2012;58(6):650-8. http://dx.doi.org/10.1590/S0104-42302012000600008. PMid:23250092. 
Anexo 1. Questões Discern Questionnaire (DQ)

1. O texto deixa claro quais são os objetivos da página?

2. O texto alcança os objetivos propostos?

3. O texto traz informações relevantes?

4. O texto deixa claro quais foram as fontes de informação consultadas pelos autores para os dados apresentados?

5. O texto deixa claro quando (data) a informação apresentada foi produzida?

6. O texto é justo, equilibrado e imparcial?

7. O texto fornece outras fontes adicionais que podem ser consultadas para o leitor obter mais informações?

8. O texto aponta que existem dúvidas/falta de consenso em relação ao tratamento?

9. O texto descreve o que ocorre passo a passo (a técnica)?

10. O texto descreve pelo menos um benefício/vantagem do tratamento?

11. O texto descreve pelo menos algum dos riscos/desvantagens do tratamento?

12. O texto descreve o que aconteceria se nenhum tratamento fosse usado?

13. O texto descreve como o tratamento afeta a qualidade de vida geral?

14. O texto deixa claro que existem outras alternativas para tratamento?

15. O texto oferece sugestões de itens ou tópicos a serem discutidos com o médico ou incentiva a discussão com o médico para que a decisão sobre o tratamento seja tomada de forma compartilhada entre o usuário e o médico?

16. Baseado nas respostas das questões anteriores, qual é a qualidade geral da publicação como fonte de informação sobre o tratamento?

Anexo 2. Questões System Usability Scale (SUS) - traduzido para o português

1. Acho que gostaria de utilizar este produto com frequência.

2. Considerei o produto mais complexo do que o necessário.

3. Achei o produto fácil de utilizar.

4. Acho que necessitaria de ajuda de um técnico para conseguir utilizar este produto.

5. Considerei que as várias funcionalidades deste produto estavam bem integradas.

6. Achei que este produto tinha muitas inconsistências.

7. Suponho que a maioria das pessoas aprenderia a utilizar rapidamente este produto.

8. Considerei o produto muito complicado de utilizar.

9. Senti-me muito confiante ao utilizar este produto.

10. Tive que aprender muito antes de conseguir lidar com este produto.

11. Você tem alguma crítica e/ou sugestão referente ao produto? (opcional). 